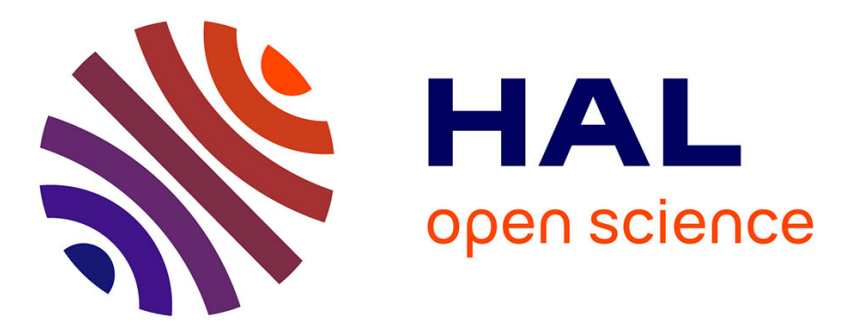

\title{
Multitasking Actors of Staphylococcus aureus Metabolism and Virulence
}

\author{
Xin Tan, Mathieu Coureuil, Alain Charbit, Anne Jamet
}

\section{To cite this version:}

Xin Tan, Mathieu Coureuil, Alain Charbit, Anne Jamet. Multitasking Actors of Staphylococcus aureus Metabolism and Virulence. Trends in Microbiology, 2020, 28 (1), pp.6-9. 10.1016/j.tim.2019.10.013 . hal-02438064

\section{HAL Id: hal-02438064 https://hal.science/hal-02438064}

Submitted on 19 Nov 2020

HAL is a multi-disciplinary open access archive for the deposit and dissemination of scientific research documents, whether they are published or not. The documents may come from teaching and research institutions in France or abroad, or from public or private research centers.
L'archive ouverte pluridisciplinaire HAL, est destinée au dépôt et à la diffusion de documents scientifiques de niveau recherche, publiés ou non, émanant des établissements d'enseignement et de recherche français ou étrangers, des laboratoires publics ou privés. 
$1 \quad$ Multitasking actors of Staphylococcus aureus metabolism and virulence

2 INSERM U1151 - CNRS UMR 8253, Institut Necker-Enfants Malades. Equipe 7:

$5 \quad$ Pathogénie des Infections Systémiques, Paris, France

$6{ }^{3}$ Service de Microbiologie, Hôpital Necker-Enfants Malades, Assistance Publique-Hôpitaux

7 de Paris, Paris, France

8 *Correspondence: anne.jamet@inserm.fr (A. Jamet).

9 Tel: 00331726065 06; Address: Institut Necker Enfants-Malades, 14 rue Maria Helena

10 Vieira Da Silva, Paris 75014, France

\section{Keywords}

Staphylococcus aureus; metabolism; virulence

\section{Abstract (50 words)}

Recent studies have uncovered the striking commonality of multitasking molecular actors linking metabolism and virulence regulation. Beyond the well-known importance of carbohydrate and amino acid metabolism regulators in coordinating metabolic pathways and pathogenesis, we highlight recent major advances linking lipid and nucleic acid pathways to virulence regulation in S. aureus. 


\section{Staphylococcus aureus virulence and metabolism}

Staphylococcus aureus, which is both a human commensal and pathogen, is mainly found in the anterior nares, but can also colonize the skin and mucosa. The bacterium is responsible for a wide variety of infections, ranging from mild skin infections to life-threatening bacteremia. When facing different host microenvironments, S. aureus must cope with nutrient shifts and modulates its virulence level by well-known interconnected regulatory systems including Agr and SaeRS. Agr system controls the switch from expression of cell-surface associated proteins, including fibronectin-binding proteins (FnBPs; FnbA and FnbB), which are involved in biofilm formation and internalization within host cells, to secreted toxins including alphahemolysin.

Multiple metabolic factors involved in carbohydrate or amino acid metabolism have been found to moonlight in virulence. We highlight here recent discoveries linking lipid and nucleobase metabolism to $S$. aureus pathogenesis.

\section{Purine and pyrimidine biosynthetic pathways}

While investigating the unusual ability of several isolates to form clumps in liquid culture (hyper-clumping phenotype), Goncheva et al. identified mutations in the purine biosynthesis repressor gene, purR [1]. They discovered that, besides its role in purine pathway regulation, PurR was also involved in virulence modulation. Indeed, purR mutants were hypervirulent in a systemic mice infection model. Hypervirulence of the purR mutants correlated to their hyper-clumping phenotype in serum, due to increased expression of genes encoding FnBPs. The clinical relevance of purR mutations is supported by the fact that purR variants are found in public databases of genomes [1]. These variants could emerge during the course of infection since Goncheva et al. were able to recover purR mutants from the organs of mice initially infected with a wild-type USA300 strain [1].

In a paper published a few months later, Sause et al. confirmed that, in a purR defective mutant, overexpression of FnbA increases bacterial burden in tissues in a murine model of bacteremia [2]. Sause et al. demonstrated direct binding of PurR to the promoters of several virulence genes (including $f n b A$, genes coding for leukocidins and alpha-hemolysin) and regulators, highlighting that multiple factors contribute to the hypervirulence of purR mutants independently of purine biosynthesis [2].

One can hypothesize that a decrease in purine availability during the course of infection requires concomitant PurR derepression of both purine biosynthesis pathway and virulence 
factor expression. Besides, as shown by Goncheva et al. [1], stress induced by the host immune response selects for purR-inactivating mutations, which likely confers metabolic advantage that incidentally locks these strains in a hypervirulence state.

A role in pathogenesis has also been recently reported for PyrR, the repressor of the pyrimidine biosynthesis operon, by Copin et al. while investigating the epidemic success of a USA300 subclone [3]. In this subclone, a pyrR-inactivating mutation increased pyr operon gene expression, providing a colonization and transmission advantage in a mouse gastrointestinal model [3].

Collectively, these findings provide novel links between $S$. aureus pathogenesis and nucleobase metabolism through modulation of virulence and colonization potential (Table 1).

The other recent major findings linking pathogenesis and metabolism in $S$. aureus pertain to the regulation of lipid pathways and more specifically highlight the key roles of fatty acids.

\section{Fatty acid (FA) metabolism}

FA metabolism has been recently linked to both virulence and colonization potential in $S$. aureus. Fatty acid kinase A (FakA encoded by fakA aka $v f r B$ ) phosphorylates and incorporates exogenous FAs into the $S$. aureus cell membrane [4]. Whereas a $S$. aureus strain lacking FakA exhibits an increased virulence in a mouse model of dermonecrosis [5], it has a virulence defect in a mouse model of bacteremia [6]. While the increased skin virulence remained unexplained, Lopez et al. correlated the virulence attenuation observed in the bacteremia model with a lack of type VII secretion system (T7SS) activation. The S. aureus T7SS contributes to virulence in murine infection models. The authors suggest that $S$. aureus modulates the expression of its T7SS in response to environmental levels of host-derived FAs. Indeed, the FakA-mediated incorporation of environmental FAs in the bacterial membrane modifies its fluidity, which could trigger T7SS expression [6].

Transcriptional profiling of a fakA mutant provided additional explanation for its virulence defect by showing severe attenuation in SaeRS-dependent virulence factor transcription [7]. The SaeRS two-component system is a master regulator of virulence genes in S. aureus. Ericson et al. demonstrated that FAs that fail to be incorporated into the membrane accumulate within fakA mutant cells and have a direct inhibitory effect on SaeRS signaling. Thus, by incorporating FAs into $S$. aureus membranes, FakA modifies both membrane fluidity and the FA intracellular pool, which impact T7SS expression and SaeRS signaling respectively. Interestingly, $f a k A$ mutations were identified in clinical isolates from cystic 
fibrosis patients [8]. Collectively, these studies suggest a key but still underappreciated key role for FakA in S. aureus pathogenesis.

Studies on bacterial lipid metabolism may further our understanding of the role of FAs in bacterial pathogenesis, since, in addition of being major energy sources and important component of membranes, lipid-related molecules released by bacteria are signal molecules that can be sensed by other bacteria and by host immune cells. Indeed, bacterial lipoproteins and lipopeptides are pathogen-associated molecular patterns (PAMPs), which are major agonists for Toll-like receptor (TLR) signaling during the innate immune response. By conducting a screen to identify S. aureus factors of immune evasion, Grayczyk et al. found that a mutation in the lipoic acid synthetase gene (lipA) elicited a hyper-inflammatory response in macrophages [9]. Lipoic acid (LA) is a fatty acid-related cofactor covalently linked to multiprotein enzymatic complexes such as pyruvate dehydrogenase (PDH), yielding lipoyl-PDH [9]. Wild type S. aureus, but not a lipA mutant, releases nanomolar quantities of lipoyl-PDH into the culture supernatant, which is able to blunt TLR2-dependent activation of macrophages. To support the immunosuppressive function of protein lipoylation, Grayczyk et al. showed that a synthetic tripeptide containing a lipoyl residue, but not the unmodified tripeptide, was sufficient to suppress TLR1/2-dependent macrophage activation by S. aureus lipoproteins [9]. The authors thus proposed that secreted lipoyl-PDH prevents lipoproteindependent activation of macrophages through its lipoyl moiety. It is likely that lipoylated proteins antagonize lipoprotein activation of macrophages by competing for TLR binding.

More recently, the predominant $S$. aureus secreted lipase, Geh, was demonstrated to also be a potent immunomodulator [10]. Besides liberating long chain FAs from host-derived lipoproteins for nutritional purpose, Geh also hydrolyzes S. aureus lipoproteins, which prevents their recognition by host immune cells and fulfills an elegant immune evasion function, which ultimately promotes persistence [10]. Thus, a protein can exhibit several functions by displaying several activities or by performing the same activity on different targets.

LipA and Geh are two striking examples of $S$. aureus enzymes involved in both lipid metabolism and immune evasion. While LipA seems to prevent lipoprotein activation of TLR, Geh prevents immune cell activation by directly hydrolyzing lipoproteins. Therefore, FakA, LipA and Geh represent multitasking enzymes that play a critical role in $S$. aureus pathogenesis in addition to their function in lipid metabolism (Table 1).

\section{Concluding Remarks}


The list of multifunctional proteins frequently referred to as "moonlighting proteins" or multitasking enzymes involved in metabolic pathways and bacterial pathogenesis is rapidly expanding. The online MoonProt Database includes around 400 proteins displaying such moonlighting functions and it is likely that many other moonlighting proteins have yet to be found (http://www.moonlightingproteins.org/). Since additional functions can be difficult to predict, multifunctional proteins are often serendipitously discovered during the course of proteomic or genomic data analysis. However, while such omics studies may help uncover unexpected new functions of proteins not previously linked to virulence, the well-known classical virulence factors are resourceful and can still surprise us. In particular, it was known that when located extracellularly, enzymes of glycolysis could contribute to bacterial adhesion. Strikingly, it is now shown that translocation of cytoplasmic proteins into the extracellular milieu by $S$. aureus requires a functional $a g r$ system through the membrane-damaging activity of phenol-soluble modulin peptides [11]. In addition, $S$. aureus biofilm proteome profiling revealed a high abundance of secreted virulence factors, including hemolysins, leukotoxins, and lipases trapped within the extracellular matrix where they fulfill an additional stabilizing function by generating electrostatic bridges [12]. Altogether, these studies underline how activities of multitasking proteins can be tightly linked with $S$. aureus pathogenicity.

Table 1. Molecular actors of metabolism with a recently described role in $S$. aureus pathogenesis.

\begin{tabular}{|l|l|l|}
\hline Gene & \multicolumn{1}{|c|}{ Metabolic function } & \multicolumn{1}{c|}{ Impact on pathogenesis } \\
\hline purR & Repression of purine biosynthesis & $\begin{array}{l}\text { Impacts fibronectin binding proteins (FnBPs), } \\
\text { leukocidins and } \alpha \text {-toxin regulation. purR } \\
\text { inactivation leads to hyper-clumping, } \\
\text { hypervirulence and increased biofilm } \\
\text { formation [1, 2]. }\end{array}$ \\
\hline pyrR & $\begin{array}{l}\text { Repression of pyrimidine } \\
\text { biosynthesis }\end{array}$ & $\begin{array}{l}\text { pyrR inactivation provides a colonization and } \\
\text { transmission advantage in a mouse } \\
\text { gastrointestinal model [3]. }\end{array}$ \\
\hline fakA & $\begin{array}{l}\text { Fatty acid phosphorylation and } \\
\text { uptake into S. aureus cell } \\
\text { membrane }\end{array}$ & $\begin{array}{l}\text { Impacts type VII secretion system activation } \\
\text { [6] and SaeRS-dependent virulence factor } \\
\text { transcription [7]. } \\
\text { fakA mutants exhibit a virulence defect in a } \\
\text { mouse model of bacteremia [6] and an }\end{array}$ \\
\hline
\end{tabular}




\begin{tabular}{|c|c|c|}
\hline & & $\begin{array}{l}\text { increased virulence in a mouse model of } \\
\text { dermonecrosis [5]. }\end{array}$ \\
\hline geh & $\begin{array}{l}\text { Hydrolysis of environmental long } \\
\text { chain lipids and } S . \text { aureus } \\
\text { lipoproteins released in the } \\
\text { extracellular milieu }\end{array}$ & $\begin{array}{l}\text { By hydrolyzing lipoprotein pathogen- } \\
\text { associated molecular patterns (PAMPs), Geh } \\
\text { prevents their recognition by host immune } \\
\text { cells and allows immune evasion [10]. }\end{array}$ \\
\hline $\operatorname{lip} A$ & $\begin{array}{l}\text { Synthesis of lipoic acid (LA), a } \\
\text { cofactor that covalently modify } \\
\text { proteins such as the pyruvate } \\
\text { dehydrogenase (PDH) complex } \\
\text { yielding lipoyl-PDH }\end{array}$ & $\begin{array}{l}\text { Lipoyl-proteins antagonize lipoprotein } \\
\text { activation of macrophage likely by a } \\
\text { competition for TLR binding [9]. }\end{array}$ \\
\hline
\end{tabular}

\section{REFERENCES}

1 Goncheva, M.I., et al. (2019) Stress-induced inactivation of the Staphylococcus aureus purine biosynthesis repressor leads to hypervirulence. Nature communications 10, 775 2 Sause, W.E., et al. (2019) The purine biosynthesis regulator PurR moonlights as a virulence regulator in Staphylococcus aureus. Proceedings of the National Academy of Sciences of the United States of America 116, 13563-13572

3 Copin, R., et al. (2019) Sequential evolution of virulence and resistance during clonal spread of community-acquired methicillin-resistant Staphylococcus aureus. Proceedings of the National Academy of Sciences of the United States of America 116, 1745-1754 4 Parsons, J.B., et al. (2014) Identification of a two-component fatty acid kinase responsible for host fatty acid incorporation by Staphylococcus aureus. Proceedings of the National Academy of Sciences of the United States of America 111, 10532-10537

5 Bose, J.L., et al. (2014) Identification of the Staphylococcus aureus vfrAB operon, a novel virulence factor regulatory locus. Infection and immunity 82, 1813-1822

6 Lopez, M.S., et al. (2017) Host-derived fatty acids activate type VII secretion in Staphylococcus aureus. Proceedings of the National Academy of Sciences of the United States of America 114, 1122311228

7 Ericson, M.E., et al. (2017) Role of Fatty Acid Kinase in Cellular Lipid Homeostasis and SaeRSDependent Virulence Factor Expression in Staphylococcus aureus. mBio 8, e00988-00917 8 Tan, X., et al. (2019) Chronic Staphylococcus aureus lung infection correlates with proteogenomic and metabolic adaptations leading to an increased intracellular persistence. Clinical infectious diseases : an official publication of the Infectious Diseases Society of America, doi: 10.1093/cid/ciz1106

9 Grayczyk, J.P., et al. (2017) A Lipoylated Metabolic Protein Released by Staphylococcus aureus Suppresses Macrophage Activation. Cell host \& microbe 22, 678-687 e679

10 Chen, X. and Alonzo, F., 3rd (2019) Bacterial lipolysis of immune-activating ligands promotes evasion of innate defenses. Proceedings of the National Academy of Sciences of the United States of America 116, 3764-3773

11 Ebner, P., et al. (2017) Non-classical Protein Excretion Is Boosted by PSMalpha-Induced Cell Leakage. Cell reports 20, 1278-1286

12 Graf, A.C., et al. (2019) Virulence Factors Produced by Staphylococcus aureus Biofilms Have a Moonlighting Function Contributing to Biofilm Integrity. Molecular \& cellular proteomics : MCP 18, 1036-1053 